\title{
Normal reference values of left ventricular volumetric parameters in healthy adults - real-life single-center experience from the three-dimensional speckle-tracking echocardiographic MAGYAR-Healthy Study
}

\author{
Árpád Kormányos, Anita Kalapos, Péter Domsik, Nándor Gyenes, Csaba Lengyel, Attila Nemes \\ Department of Medicine, Medical Faculty, Albert Szent-Györgyi Clinical Center, University of Szeged, Szeged, Hungary \\ Correspondence to: Attila Nemes, MD, PhD, DSc, FESC. Department of Medicine, Medical Faculty, Albert Szent-Györgyi Clinical Center, University \\ of Szeged, H-6725 Szeged, Semmelweis street 8, Hungary. Email: nemes.attila@med.u-szeged.hu.
}

\begin{abstract}
Background: The left ventricle (LV) is the pump of the peripheral circulation, therefore its non-invasive accurate volumetric and functional assessment is essential. Three-dimensional (3D) speckle-tracking echocardiography (STE) is a new tool with capability of quantification of LV volumes and ejection fraction (EF). In the present study, age- and gender-dependency of 3DSTE-derived LV volumetric parameters were aimed to be quantified in healthy adults.

Methods: The present study involved 298 healthy adults. Data acquisition took place over a 6 year period (2011-2017), during which 123 adults had to be excluded due to inferior image quality. The study population was further divided into 4 groups based on age decades. In every case, a complete two-dimensional echocardiography was performed followed by 3DSTE examination.

Results: No significant differences were detected between the different age groups regarding 3D enddiastolic volume (EDV) and 3D end-systolic volume (ESV) and their body surface area (BSA)-indexed counterpart. 3DEF differed significantly between the 30-39-year-old males and 40-49-year-old males $(\mathrm{P}=0.04)$. Between the youngest and oldest patient group, only $3 \mathrm{D}$ left ventricular mass (LVM) was significantly different $(\mathrm{P}=0.02)$. The $18-29$-year-old females had a significantly different 3DLVM $(\mathrm{P}<0.001)$ compared to that of the 50+ year-old females. Between the 40-49-year-old and 50+ year-old females 3DLVM $(\mathrm{P}=0.02)$ and BSA-indexed 3DLVM $(\mathrm{P}=0.05)$ proved to be significantly different.
\end{abstract}

Conclusions: 3DSTE seems to be a reasonably viable tool for the quantification of LV volumetric parameters.

Keywords: Healthy; left ventricular volume; three-dimensional; echocardiography

Submitted Jun 22, 2020. Accepted for publication Nov 10, 2020.

doi: 10.21037/qims-20-786

View this article at: http://dx.doi.org/10.21037/qims-20-786

\section{Introduction}

The left ventricle (LV) is the pump of the peripheral circulation, therefore its non-invasive accurate volumetric and functional assessment is essential (1). With its widespread availability, two-dimensional (2D) echocardiography (2DE) plays an important role in the assessment of conventional LV parameters including diameters, volumes and ejection fraction (EF) from typical views (2). However, $2 \mathrm{DE}$ is known to have significant limitations in measuring these parameters (3). Threedimensional (3D) speckle-tracking echocardiography (STE) is a relatively new tool which is capable of quantifying $\mathrm{LV}$ volumes and EF (4). Previously it has been validated by several accepted methodologies such as sonomicrometry, cardiac magnetic resonance imaging (CMR), 
conventional 2DE, 2DSTE and volumetric real-time 3D echocardiography (RT3DE) (1,5-9). To the best of the authors' knowledge, limited number of studies employing 3DSTE is available analyzing $\mathrm{LV}$ volumetric parameters and EF in real-life settings, therefore age- and genderdependency of 3DSTE-derived LV volumetric parameters were aimed to be quantified in healthy adults.

\section{Methods}

\section{Patient population}

The present study involved 298 healthy adults. A volunteer was considered to be healthy, if he/she had no history of chronic illness, no history of medication use, had a negative physiological examination and routine electrocardiography and echocardiography showing normal parameters. Data acquisition took place over a 6-year period (2011-2017), during which 123 adults had to be excluded due to inferior image quality. The study population was further divided into 4 groups based on age decades. The present study is a part of the MAGYAR-Healthy Study (Motion Analysis of the heart and Great vessels bY three-dimensionAl speckletRacking echocardiography in Healthy subjects), where 'magyar' means 'Hungarian' in the Hungarian language. This 3DSTE study has been created to assess and determine physiological 3DSTE-derived volumetric and functional parameters of the heart. All subjects gave informed consent, the institutional human research committee approved the study and it also complied with the guidelines set by the Declaration of Helsinki.

\section{Two-dimensional echocardiography}

The 2DE data acquisition was carried out using a Toshiba Artida $^{\mathrm{TM}}$ imaging system (Toshiba Medical Systems, Tokyo, Japan) and a PST-30SBP (1-5 MHz) phased-array transducer. Experienced operators performed complete 2DE examinations in all subjects (DP, AK, ÁK, NG) together with a Doppler study according to the current clinical standards (2,10-12). In all cases, left atrial diameter, LV dimensions, volumes and EF were measured. To determine the severity of valvular regurgitations, a subjective visual scale was used, where 0 means no regurgitation and 4 represents severe regurgitation. Doppler echocardiography was used for exclusion of valvular stenosis.

\section{Three-dimensional speckle-tracking echocardiography}

For 3DSTE measurements, the same Toshiba Artida ${ }^{\mathrm{TM}}$ echocardiographic equipment (Toshiba Medical Systems, Tokyo, Japan) was used with a PST-25SX matrix-array transducer (Toshiba Medical Systems, Tokyo, Japan) (1). Data acquisition was carried out from the apical window during a single breath-hold. A full volume pyramid shape dataset was made up of 6 smaller wedge-shaped subvolumes, which were recorded during 6 constant RR intervals. For offline image analysis purposes, the vendor-supplied 3D Wall Motion Tracking software (version 2.7) was used. During analysis, the apical four- and two-chamber views were automatically selected, while the three cross-sectional planes were manually specified by the operator. Following LV endocardial border detection, the software created a virtual $3 \mathrm{D}$ model of the $\mathrm{LV}$, which then was used to compute $\mathrm{LV}$ volumetric parameters as well as different $\mathrm{LV}$ strain parameters together with $\mathrm{LV}$ rotational parameters (Figure 1). During a rigorous quality control, all dataset was excluded where not all $16 \mathrm{LV}$ segments could be visualized.

The following 3DSTE-derived parameters were assessed:

- LV end-diastolic and end-systolic volume (3DEDV and $3 \mathrm{DESV}$, respectively);

* LV ejection fraction (3DEF);

* LV mass (3DLVM) (as assessed automatically by the software following automatic contour detection).

\section{Statistical analysis}

All data are reported as mean \pm standard deviation. $\mathrm{P}$ values $<0.05$ were considered to be statistically significant. Fischer's exact test was used for categorical variables. In case of continuous variables, Shapiro-Wilks test was performed firstly to test normality of distribution, then the homogeneity of variance was assessed using Levene's test. For normally distributed continuous variables, Student's $t$-test was used. For variables not following normal distribution, Mann-Whitney-Wilcoxon test was applied. RStudio was used for statistical analysis (RStudio Team (2015). RStudio: Integrated Development for R. RStudio, Inc., Boston, MA). For offline data analysis MATLab was used (The MathWorks Inc, Natick, Massachusetts).

\section{Results}

\section{Demographic and two-dimensional echocardiographic data}

The study population has been broken up into four subgroups based on their age: $18-29$ years (mean age: $23.6 \pm 2.8$ years, 45 males), 30-39 years (mean age: 

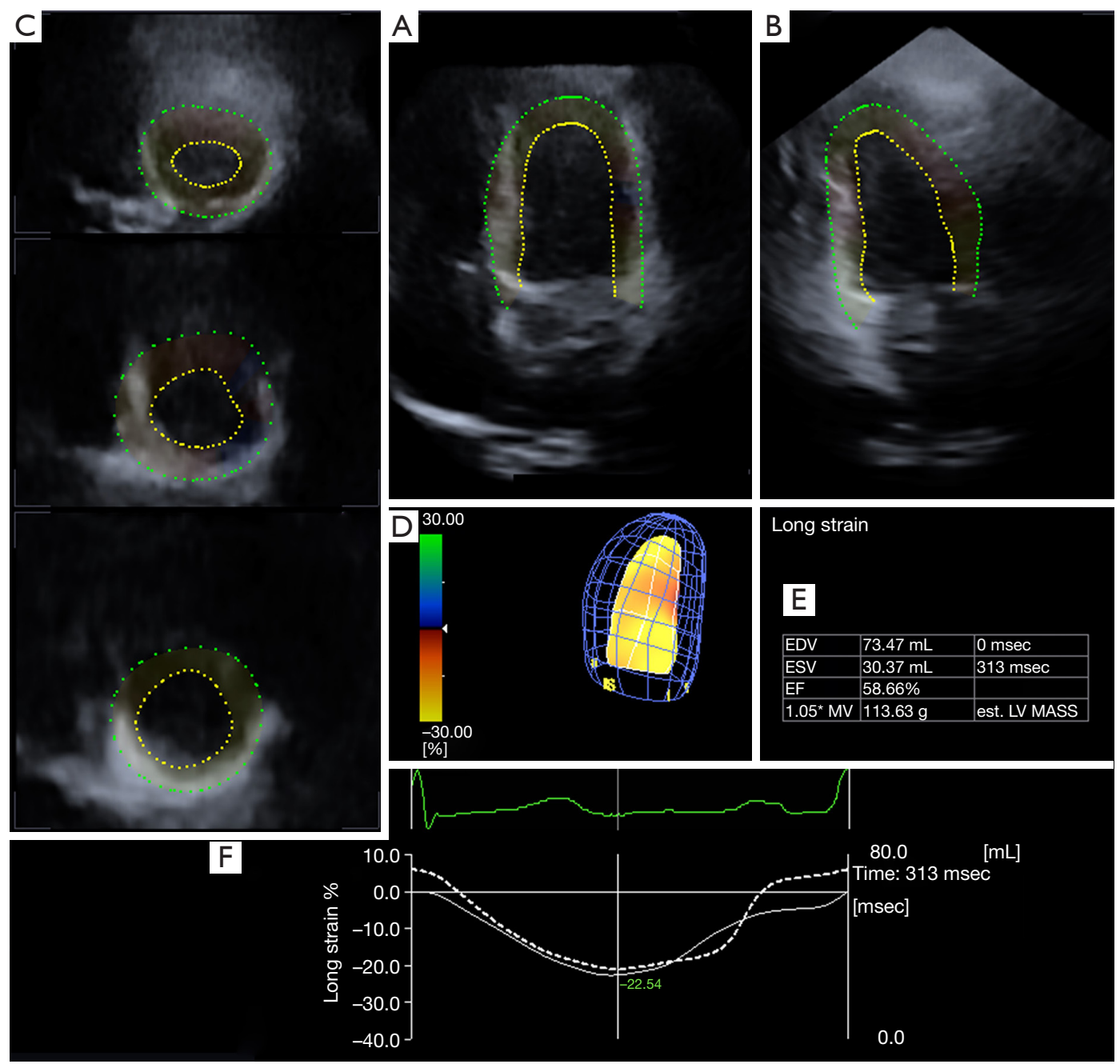

Figure 1 Three-dimensional (3D) speckle-tracking assessment of left ventricular (LV) volumes and mass is presented. 'A' shows the apical four-chamber view, 'B' shows the apical two-chamber view of the $L V$, while 'C3-5' are cross sections of the LV. 'D' shows the 3D cast of the $\mathrm{LV}$ and ' $\mathrm{E}$ ' shows $\mathrm{LV}$ volumetric and mass data corresponding to the measurement. ' $\mathrm{F}$ ' shows the actual $\mathrm{LV}$ volume change during a cardiac cycle (dashed line). LV, left ventricle; RV, right ventricle; LA, left atrium; RA, right atrium.

$33.5 \pm 2.7$ years, 27 males), $40-49$ years (mean age: $43.1 \pm 3.2$ years, 11 males) and $50+$ years (mean age: $56.1 \pm 4.4$ years, 12 males). The results of $2 \mathrm{DE}$ and Doppler study are shown in Table 1. None of the volunteers had higher than grade 1 valvular regurgitation or significant valvular stenosis.

\section{DSTE-derived $L V$ volumetric data}

No significant differences were detected between the different age groups regarding $3 \mathrm{DEDV}$ and $3 \mathrm{DESV}$ and their BSA-indexed counterpart. 3DEF differed significantly between the 30-39-year-old males and 40-49-year-old males $(\mathrm{P}=0.04)$. Between the youngest and oldest patient groups, only $3 \mathrm{DLVM}$ was significantly different $(\mathrm{P}=0.02)$. The 18-29-year-old females had a significantly different 3 DLVM $(\mathrm{P}<0.001)$ compared to that of the $50+$ yearold females. Between the 40-49-year-old and 50+ yearold females, 3DLVM $(\mathrm{P}=0.02)$ and BSA-indexed 3DLVM $(\mathrm{P}=0.05)$ proved to be significantly different (Table 2).

\section{Gender differences in 3DSTE-derived $L V$ volumetric data}

In the age group of $18-29$ years, 3DEDV $(\mathrm{P}<0.001)$, 
Table 1 Demographic and 2D echocardiographic data of enrolled healthy volunteers

\begin{tabular}{|c|c|c|c|}
\hline & All subjects $(n=175)$ & Males $(n=95)$ & Females $(n=80)$ \\
\hline Age (years) & $32.7 \pm 12.3$ & $33.0 \pm 10.9$ & $32.4 \pm 13.7$ \\
\hline Male gender (\%) & $95(54)$ & $95(100)$ & $0(0)$ \\
\hline $\operatorname{BSA}\left(m^{2}\right)$ & $1.85 \pm 0.11$ & $1.95 \pm 0.18$ & $1.67 \pm 0.23$ \\
\hline Diabetes mellitus (\%) & $0(0)$ & $0(0)$ & $0(0)$ \\
\hline Hyperlipidaemia (\%) & $0(0)$ & $0(0)$ & $0(0)$ \\
\hline \multicolumn{4}{|l|}{ Two-dimensional echocardiography } \\
\hline LA diameter (mm) & $36.6 \pm 4.0$ & $38.3 \pm 3.1$ & $34.7 \pm 4.1$ \\
\hline LV end-systolic diameter (mm) & $32.6 \pm 7.4$ & $32.7 \pm 2.9$ & $30.8 \pm 3.5$ \\
\hline LV end-systolic volume (mL) & $36.4 \pm 9.2$ & $40.2 \pm 8.7$ & $31.9 \pm 7.5$ \\
\hline Interventricular septum (mm) & $9.0 \pm 1.5$ & $9.5 \pm 1.3$ & $8.3 \pm 1.5$ \\
\hline LV posterior wall (mm) & $9.1 \pm 1.6$ & $9.5 \pm 1.4$ & $8.6 \pm 1.8$ \\
\hline LV ejection fraction (\%) & $65.9 \pm 4.9$ & $64.8 \pm 4.2$ & $67.2 \pm 5.4$ \\
\hline $\mathrm{E}(\mathrm{cm} / \mathrm{s})$ & $80.2 \pm 17.5$ & $77.8 \pm 16.5$ & $83.4 \pm 18.4$ \\
\hline $\mathrm{A}(\mathrm{cm} / \mathrm{s})$ & $64.6 \pm 19.7$ & $59.7 \pm 15.7$ & $70.1 \pm 22.2$ \\
\hline$E / A$ & $1.2 \pm 0.6$ & $1.3 \pm 0.5$ & $1.1 \pm 0.7$ \\
\hline
\end{tabular}

BSA, body surface area; LA, left atrium; LV, left ventricle; E, early transmitral flow velocity; A, late transmitral flow velocity.

3DESV $(\mathrm{P}<0.001)$ and 3DLVM $(\mathrm{P}<0.001)$ proved to be significantly different between the genders. In the age group of 30-39 years, 3DEDV ( $\mathrm{P}=0.004)$, 3DESV $(\mathrm{P}=0.04)$, 3DLVM $(\mathrm{P}=0.04)$ differed significantly between males and females. In the group of 40-49-year-old subjects, 3DESV $(\mathrm{P}=0.003)$ and $3 \mathrm{DLVM}(\mathrm{P}=0.02)$ were significantly different between the genders (Table 2).

\section{Feasibility of 3DSTE-derived LV quantification}

During the acquisition period (2011-2017), the experience of the operators grew significantly, thus increasing the feasibility of the 3DSTE method. For the overall duration of data acquisition, 175 measurements out of 298 proved to be acceptable (59\% success ratio). For the last year of acquisition, 46 measurements out of 58 were successful (79\% success ratio), this is a significant increase $(\mathrm{P}=0.001)$.

\section{Discussion}

Nowadays, 2DE is routinely available in the clinical practice, it is accessible in all cardiology centers all over the world. Through standardized acquisition and analysis, all 2DE-derived parameters are comparable no matter in which center the examination took place. A few of the most important of these $\mathrm{LV}$ parameters are EDV, ESV and EF. EF has been accepted as the most reliable LV systolic functional parameter with robust prognostic implications, almost every subspecialty strongly relies on it in the daily practice. There are several formulas to calculate LVEF using 2DE, the modified Simpson's formula and the area-length method are suggested to be used by the guidelines. However, both methods approximate LVEF through different mathematical equations using geometric assumptions. Furthermore, they heavily rely on the observer 


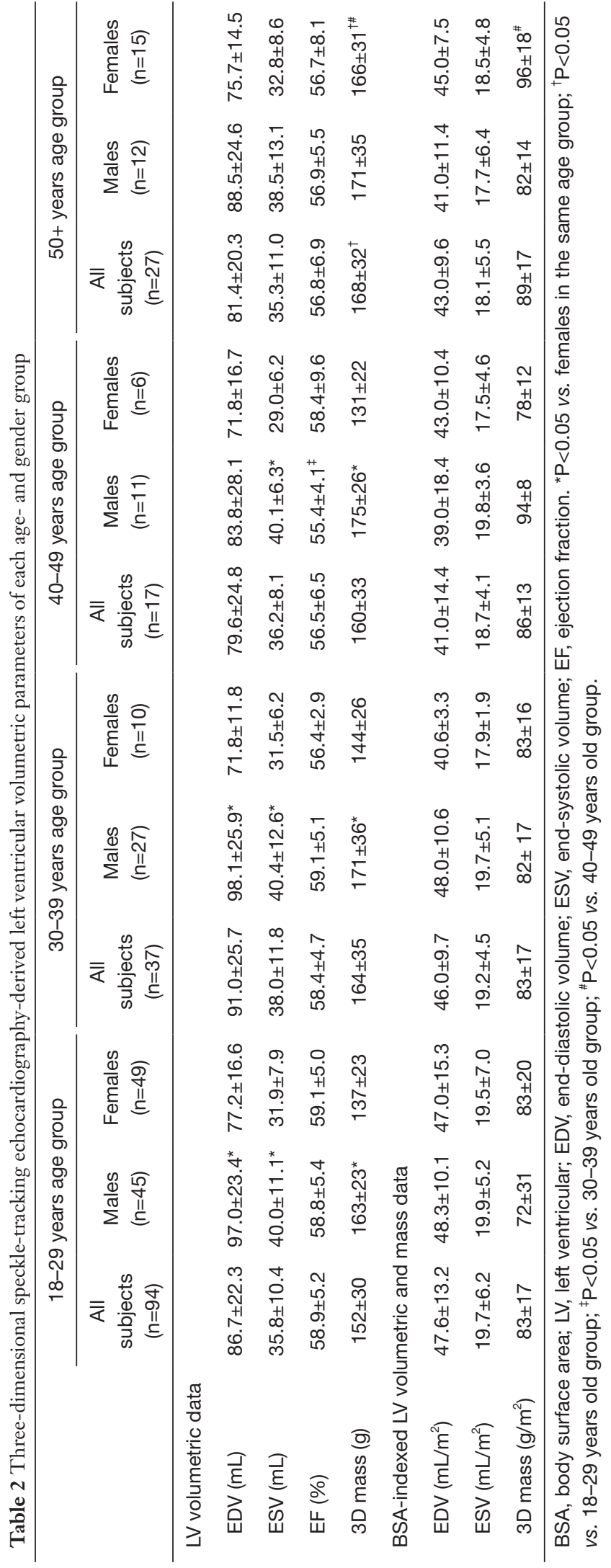

and acquisition technique resulting in a relatively inferior interobserver variability $(2,13)$.

The introduction of RT3DE and 3DSTE may be able to solve the aforementioned problems. 3DSTE is a validated, non-invasive tool in assessing $L V$ volumetric and strain parameters together with $\mathrm{LV}$ rotational variables from a single dataset $(1,5-7,14)$. During a 3 DSTE measurement, an LV model is created through endocardial tracking in $3 \mathrm{D}$ space, resulting in precise, real volumetric chamber quantification, not just approximation through a mathematical formula. However, it is worthy to note that LVEF calculated during 3DSTE measurements are somewhat lower compared to conventional $2 \mathrm{D}$ measurements. The explanation for this is that $3 \mathrm{DSTE}$ underestimates $L V$ volumetric parameters, $3 \mathrm{DEDV}$ is more affected than 3DESV (9), thus resulting in a lower 3DEF value. Mor-Avi et al. found that this underestimation of volume is the result of the lower spatial resolution of $3 \mathrm{DE}$ methodologies compared to CMR. The image quality is insufficient to clearly differentiate endocardial trabeculae, thus lumping them together with the myocardium resulting in lower $\mathrm{LV}$ volumes (15).

In a recently published paper from Takeuchi et al., a comparably large group of healthy adults $(n=313)$ were examined employing RT3DE to assess age- and genderdependency of LV and left atrial coupling. The healthy volunteers were divided according to age decades, then RT3DE-derived LV volumetric parameters were measured. According to the results, males had a significantly higher BSA-indexed 3DEDV and 3DESV compared to females, but $3 \mathrm{DEF}$ tended to be higher in females than in males. Through the age decades, 3DEDV and 3DESV steadily decreased in the higher age decades (16). In another paper from Kleijn et al., 303 healthy adults were examined using a Toshiba Artida system with 3DSTE capabilities. In this article, only the gender dependency of 3DSTE-derived LV volumetric parameters was described. 3DEDV, 3DESV and 3DLVM were found to be significantly higher in males, whereas $3 \mathrm{DEF}$ was significantly higher in females compared to the other gender (17).

The results presented in this study corroborate the aforementioned findings in a relatively large number of healthy volunteers from a single center. It has been found that 3DEDV and 3DESV are tendentiously larger in males compared to females in an age group, sometimes reaching the level of significance. These differences could also be observed in case of BSA-indexed 3DEDV and 3DESV, although to a smaller degree. The $3 \mathrm{DEF}$ was tendentiously 
higher in females compared to the other gender in a given age-group. 3DEDV and 3DESV slightly increased in the age group 30-39-year-old subjects compared to the youngest age-group, then a steady decline could be observed in these volumetric parameters. If $3 \mathrm{DEDV}$ and $3 \mathrm{DESV}$ were BSA-indexed, a slow decline could be seen with aging. Changes in LVM with aging and gender-dependency were also seen.

Results of the present study are consistent with previous non-3DSTE-derived findings demonstrating that $\mathrm{EF}$ in women is significantly higher compared to matched male counterparts, primarily due to smaller indexed EDV. Since indexed EDV is linearly correlated with indexed ESV, indexed EDV values are usually larger in men than in women (18). Moreover, gradual decline of $L V$ volumes with aging and gender- and age-dependency of LVM could also be demonstrated.

The physiological basis of these findings-over the technical reasons-has been debated for decades, it is widely accepted that cardiovascular changes occur during aging including loss of myocytes, increased stiffness of the vascular bed, neurohormonal activation etc. (19-22). The age-related decrease of $\mathrm{LV}$ volumetric parameters is partly responsible for the physiologic $\mathrm{LV}$ concentric remodelling balanced by the increase of LVEF (23). These changes might occur in response to increased blood pressure and afterload. The continuous fluid loss of the body throughout aging might also play a role (23-25). Further validation studies are warranted to better understand $\mathrm{LV}$ volumetric changes during aging.

\section{Limitation section}

* Large number of cases were excluded due to inferior image quality. 3DSTE has a relatively low temporal and spatial resolution compared to 2DSTE, furthermore since the full volume dataset is the result of the combination of 6 cardiac cycles, a socalled "stitching noise" might occur at the subvolume borders. Moreover, the effect of motion artefacts could not be excluded either.

* In the present study only LV volumetric parameters are presented. Analyzing the LV strain and rotational parameters was not the goal of the present article.

* Comparison of 3DSTE-derived LV volumetric parameters and 2DE-derived ones was not aimed in this study.
* We have not aimed to examine inter- and intraobserver variability of 3DSTE-derived LV volumetric parameters in this study.

* The present paper was not aimed to be a validation study for using 3DSTE in quantifying $\mathrm{LV}$ volumetric parameters.

* 3DLVM is higher in our study compared to other studies because LVM was calculated based on the 3D cast created by the automatic software.

\section{Conclusions}

3DSTE seems to be a reasonably viable tool for the quantification of $L V$ volumetric parameters. 3DEDV and 3DESV and their BSA-indexed counterparts show a steady decline with aging, whereas aging has no effect on $3 \mathrm{DEF}$. In a given age decade, males have higher $\mathrm{LV}$ volumetric parameters compared to females, however females have a tendentiously higher 3DEF compared to males.

\section{Acknowledgments}

Funding: None.

\section{Footnote}

Conflicts of Interest: All authors have completed the ICMJE uniform disclosure form (available at http://dx.doi. org/10.21037/qims-20-786). AN serves as an unpaid editorial board member of Quantitative Imaging in Medicine and Surgery. The authors have no conflicts of interest to declare.

Ethical Statement: All subjects gave informed consent, the institutional human research committee approved the study and it also complied with the guidelines set by the Declaration of Helsinki.

Open Access Statement: This is an Open Access article distributed in accordance with the Creative Commons Attribution-NonCommercial-NoDerivs 4.0 International License (CC BY-NC-ND 4.0), which permits the noncommercial replication and distribution of the article with the strict proviso that no changes or edits are made and the original work is properly cited (including links to both the formal publication through the relevant DOI and the license). See: https://creativecommons.org/licenses/by-nc-nd/4.0/. 


\section{References}

1. Nemes A, Forster T. Recent echocardiographic examination of the left ventricle - from M-mode to 3D speckle-tracking imaging. Orv Hetil 2015;156:1723-40.

2. Lang RM, Badano LP, Mor-Avi V, Afilalo J, Armstrong A, Ernande L, Flachskampf FA, Foster E, Goldstein SA, Kuznetsova T, Lancellotti P, Muraru D, Picard MH, Rietzschel ER, Rudski L, Spencer KT, Tsang W, Voigt JU. Recommendations for cardiac chamber quantification by echocardiography in adults: an update from the American Society of Echocardiography and the European Association of Cardiovascular Imaging. J Am Soc Echocardiogr 2015;28:1-39.e14.

3. Shimada YJ, Shiota T. A meta-analysis and investigation for the source of bias of left ventricular volumes and function by three-dimensional echocardiography in comparison with magnetic resonance imaging. Am J Cardiol 2011;107:126-38.

4. Seo Y, Ishizu T, Atsumi A, Kawamura R, Aonuma K. Three-dimensional speckle tracking echocardiography. Circ J 2014;78:1290-301.

5. Nesser HJ, Mor-Avi V, Gorissen W, Weinert L, Steringer-Mascherbauer R, Niel J, Sugeng L, Lang RM. Quantification of left ventricular volumes using three-dimensional echocardiographic speckle tracking: comparison with MRI. Eur Heart J 2009;30:1565-73.

6. Kleijn SA, Brouwer WP, Aly MF, Rüssel IK, de Roest GJ, Beek AM, van Rossum AC, Kamp O. Comparison between three-dimensional speckle-tracking echocardiography and cardiac magnetic resonance imaging for quantification of left ventricular volumes and function. Eur Heart J Cardiovasc Imaging 2012;13:834-9.

7. Kleijn SA, Aly MF, Terwee CB, van Rossum AC, Kamp O. Reliability of left ventricular volumes and function measurements using three-dimensional speckle tracking echocardiography. Eur Heart J Cardiovasc Imaging 2012;13:159-68.

8. Ashraf M, Myronenko A, Nguyen T, Inage A, Smith W, Lowe RI, Thiele K, Kroeker CAG, Tyberg JV, Smallhorn JF, Sahn DJ, Song X. Defining left ventricular apex-tobase twist mechanics computed from high-resolution $3 \mathrm{D}$ echocardiography: validation against sonomicrometry. JACC Cardiovasc Imaging 2010;3:227-34.

9. Driessen MMP, Kort E, Cramer MJM, Doevendans PA, Angevaare MJ, Leiner T, Meijboom FJ, Chamuleau SAJ, Sieswerda GTJ. Assessment of LV ejection fraction using real-time $3 \mathrm{D}$ echocardiography in daily practice: direct comparison of the volumetric and speckle tracking methodologies to CMR. Neth Heart J 2014;22:383-90.

10. Harkness A, Ring L, Augustine DX, Oxborough D, Robinson S, Sharma V; Education Committee of the British Society of Echocardiography. Normal reference intervals for cardiac dimensions and function for use in echocardiographic practice: a guideline from the British Society of Echocardiography. Echo Res Pract 2020;7:G1-G18.

11. Zhang Y, Sun H, Li M, Luo M, Xu F, Shao Y, Xu D. Impact of stand-alone minimally invasive radiofrequency ablation with left atrial appendectomy on left atrial function assessed by echocardiography. Quant Imaging Med Surg 2020;10:970-8.

12. Oberhoffer FS, Abdul-Khaliq H, Jung AM, Zemlin M, Rohrer TR, Abd El Rahman M. Assessment of left ventricular myocardial work in Turner syndrome patients: insights from the novel non-invasive pressure-strain loop analysis method. Quant Imaging Med Surg 2020;10:15-25.

13. Feigenbaum H. Echocardiography. 5th ed. Philadelphia; London: Lea \& Febiger; 1994. xii, 695 pp.

14. Zhou Z, Ashraf M, Hu D, Dai X, Xu Y, Kenny B, Cameron B, Nguyen T, Xiong L, Sahn DJ. Threedimensional speckle-tracking imaging for left ventricular rotation measurement: an in vitro validation study. J Ultrasound Med 2010;29:903-9.

15. Mor-Avi V, Jenkins C, Kuhl HP, Nesser HJ, Marwick T, Franke A, Ebner C, Freed BH, Steringer-Mascherbauer R, Pollard H, Weinert L, Niel J, Sugeng L, Lang RM. Real-time 3-dimensional echocardiographic quantification of left ventricular volumes: multicenter study for validation with magnetic resonance imaging and investigation of sources of error. JACC Cardiovasc Imaging 2008;1:413-23.

16. Takeuchi M, Kitano T, Nabeshima Y, Otsuji Y, Otani K. Left ventricular and left atrial volume ratio assessed by three-dimensional echocardiography: Novel indices for evaluating age-related change in left heart chamber size. Physiol Rep 2019;7:e14300.

17. Kleijn SA, Pandian NG, Thomas JD, Perez de Isla L, Kamp O, Zuber M, Nihoyannopoulos P, Forster T, Nesser HJ, Geibel A, Gorissen W, Zamorano JL. Normal reference values of left ventricular strain using threedimensional speckle tracking echocardiography: results from a multicentre study. Eur Heart J Cardiovasc Imaging 2015;16:410-6.

18. Kerkhof PLM, Peace RA, Macfairlane PW. Sex- and AgeRelated Reference Values in Cardiology, with Annotations 
and Guidelines for Interpretation. Adv Exp Med Biol 2018;1065:677-706.

19. Kaku K, Takeuchi M, Otani K, Sugeng L, Nakai H, Haruki N, Yoshitani H, Watanabe N, Yoshida K, Otsuji Y, Mor-Avi V, Lang RM. Age- and gender-dependency of left ventricular geometry assessed with real-time threedimensional transthoracic echocardiography. J Am Soc Echocardiogr 2011;24:541-7.

20. Fukuda S, Watanabe H, Daimon M, Abe Y, Hirashiki A, Hirata K, Ito H, Iwai-Takano M, Iwakura K, Izumi C, Hidaka T, Yuasa T, Murata K, Nakatani S, Negishi K, Nishigami K, Nishikage T, Ota T, Hayashida A, Sakata K, Tanaka N, Yamada S, Yamamoto K, Yoshikawa J. Normal values of real-time 3-dimensional echocardiographic parameters in a healthy Japanese population: the JAMP3D Study. Circ J 2012;76:1177-81.

21. Nikitin NP, Loh PH, de Silva R, Witte KKA, Lukaschuk EI, Parker A, Farnsworth TA, Alamgir FM, Clark AL, Cleland JGF. Left ventricular morphology, global and

Cite this article as: Kormányos Á, Kalapos A, Domsik P, Gyenes N, Lengyel C, Nemes A. Normal reference values of left ventricular volumetric parameters in healthy adultsreal-life single-center experience from the three-dimensional speckle-tracking echocardiographic MAGYAR-Healthy Study. Quant Imaging Med Surg 2021;11(4):1496-1503. doi: 10.21037/ qims-20-786 longitudinal function in normal older individuals: a cardiac magnetic resonance study. Int J Cardiol 2006;108:76-83.

22. Fiechter M, Fuchs TA, Gebhard C, Stehli J, Klaeser B, Stähli BE, Manka R, Manes C, Tanner FC, Gaemperli O, Kaufmann PA. Age-related normal structural and functional ventricular values in cardiac function assessed by magnetic resonance. BMC Med Imaging 2013;13:6.

23. Muraru D, Badano LP, Peluso D, Dal Bianco L, Casablanca S, Kocabay G, Zoppellaro G, Iliceto S. Comprehensive analysis of left ventricular geometry and function by three-dimensional echocardiography in healthy adults. J Am Soc Echocardiogr 2013;26:618-28.

24. Cheng S, Fernandes VR, Bluemke DA, McClelland RL, Kronmal RA, Lima JA. Age-related left ventricular remodeling and associated risk for cardiovascular outcomes: the Multi-Ethnic Study of Atherosclerosis. Circ Cardiovasc Imaging 2009;2:191-198.

25. Lakatta EG. Cardiovascular regulatory mechanisms in advanced age. Physiol Rev 1993;73:413-67. 